\title{
LVI. Observations and advices respecting improvements compatible with the quickest manufacture of Muscovado sugar, and conducive to the melioration of the rum
}

\section{Bryan Higgins M. D.}

To cite this article: Bryan Higgins M. D. (1806) LVI. Observations and advices respecting improvements compatible with the quickest manufacture of Muscovado sugar, and conducive to the melioration of the rum , Philosophical Magazine Series 1, 24:96, 308-322, DOI: 10.1080/14786440608563388

To link to this article: http://dx.doi.org/10.1080/14786440608563388

$$
\text { 曲 Published online: } 18 \text { May } 2009 .
$$

Submit your article to this journal ए

\section{山 Article views: 2}

Q View related articles ¿ 


\section{[ 308 ]}

LVI. Observations and Advices respecting Improvements compatille with the quickest Manufacture of Muscovado Sugar, and conducive to the Melioration of the Rum. By Bryan Higgins, $M . D$.*

Sound cane-juice consists of water, sugar, deliquescent sweet, herbaceous matter, carbonic acid, and melasses acid: and some juices contain variable quantities of other ingredients, which are not yet to be noticed.

In these pharmacentic ingredients subsist the primary or chemical principles of many vegetable acids. But experience shows, that the composition of attractive forces, resulting from such proportions of the principles as take place in the recent juice, tend chiefly to the formation of an acid sinilar to vinegar, and of an additional quantity of carbonic acid and melasses acid.

For in the course of 12 or 18 hours the juice mantles by the rise and escape of carbonic acid in the elastic state: at an earlier period it smells sour or acetous : and by the effect of such delay on the sugar producible from it, it is certain that there is an addition to the original quantity of the melasses acid.

This last is the ingredient which most powerfully impedes the crystallization and separation of the saccharine matter from the deliquescent sweet and mother-liquor called melasses. As it lessens the quantity of saccharine crystals, and increases that of melasses mothe--liquor; and as it is highly probable that melasses contain the like acid as a constituent principle, I give it the temporary name of melasses acid.

Herbaceous matter is that of which some part shows itself in the yawing, and more in the boiling of juice which had been cleared from gross filth by filtration. It is that which we endeavour to separate from the saccharine liquor by yawing and skimming.

The herbaceous matter has some analogy to gummy resins, but has a much nearer similitude, in chemical character, to the dregs of refined indigo, or that vegetable sub-

- From Part I. of Dr. Higgins's Observations, \&c. published in Jamaica. 
stance which constitutes the chief difference between the finest and the basest indigo.

The herbaceous matter of cane-juice, like that of indigo, varies with the constitution of the plant in different soils and seasons, and especially in respect to its solubility; insomuch that some juices hold about $\frac{1}{300} \mathrm{dth}$ part of it in strict solution after boiling, while others bold not looodth.

But as herbaceous matter is rendered more soluble by the intervention of carbonic acid, any cane-juice holds more herbaceous matter in solution before it has been heated, than it can retain at the temperature of yawing or boiling.

For in the augmented temperature the carbonic acid forsakes the herbaceous matter to combine with that which makes the acid aëriform; the minute gaseous bubbles in their escape agitate and impel the particles lately thrown out of solution, until in their coalescence they become not only visible but large. We may express this change in the clear recent juice by the agency of fire alone, as the workmen do, by saying the liquor breaks.

Fresh cane-juice begins to break when the heat approaches to 140 degrees of Fahrenheit; and the herbaceous matter, which has felt no greater heat, has an olive green colour.

Whether this be exposed to greater heat, or we advert to that which is thrown out during the subsequent reduction of the juice to sugar, the herbaceous matter is found to change colour with the increase of temperature, through gradations of yellow, olive, and brown, increasing in intensity and darkness until the matter is charred to blackness. As it changes in colour it becomes less soluble. The carbonic acid continues to escape, and the extricated herbaceous matter accumulates to the surface, while the liquor is heated to $195^{\circ}$.

Now the watery vapour arising with the carbonic acid bubbles, pushes the cleansed liquor frothing white through intervals in the swollen scum. This, which is called yawing, shows that a greater heat would cause a boiling commotion: but if some time be allowed for the residuary carbonic acid to escape, the liquor will not boil until this heat amounts to $206^{\circ}$, or within five degrees of the heat of boiling water. 
The aëriform bubbles entangled in the herbaceous matter render it more buoyant than it would otherwise be, and enable it to carry with it, and to sustain at the surface, any accidental filth of the liquor: and the scum thus produced is by its own nature sufficiently tenacious to be separable by the skimmer, or by drawing away the depurated juice. from beneath it.

But, if the buoying bubbles be expelled by greater heat and the commotion of boiling, the scum will be broken into the liquor.

The skimmer will now avail nothing; but the herbaceous matter, once thrown out of solution, will subside with the filth, in an hour, in a cooling quiescent liquor, and will leave it transparent, although it still retain that quantity of herbaceous matter, which the water, with the last adherent portions of carbonic acid, can dissolve.

But as this depuration by subsidence cannot be awaited without injury to the juice, the foul scum ought to be removed before the liquor boils.

In consequence of this limited solubility, the residuary herbaceous matter becomes extricated afterwards in quantity proportionate to that of the watery solvent which is expelled by evaporation, and the reduced liquor becomes turbid by the extricated herbaceous particles.

However often the process of evaporation is stopped, and the liquor is depurated to perfect transparency, by subsidence or otherwise, it will become turbid again, by the deposition of herbaceous dregs, when the evaporation is renewed; and it will thus yicld drogs to the end; or until the residuary liquor becomes so far saturated with sugar as to be incapable of holding the less soluble herbaceous matter in solution.

All this takes place whether a moderate dose of temper be used or not. But the cleansing by subsidence is quickest when temper is used.

To ascertain the true use of temper, we must advert to its agency on herbaceous matter, carbonic acid, and melasses acid.

Lime powerfully attracts carbonic acid: and although 
lime be a soluble body, and although it meet the acids in the aëriform state, it forms with it quickly an insoluble body similar to whiting or chalk.

Lime also combines with a triple or quadruple quantity of herbaceous matter to form a compound less soluble than the latter in water; and in cane-juice, lime meeting carbonic acid and herbaceous matter, unites with both to form a triple compound. For if the lime used in clarifiers were to unite with the carbonic acid only, we should find bottoms consisting of whiting, which I have looked for, but could never obtain.

It is by virtue of these relations that a small quantity of lime, or transparent lime water in which the lime can be only $\frac{1}{5-0}$ th part of the whole, when added to cane-juice that has been duly cleared, renders it presently turbid with herbaceous matter now extricated, and thus facilitates the abstraction of this matter by subsidence. Thus, also, canejuice which is a little wheyey or clouded is broken to flocculence by transparent lime water as well as by lime.

I say the liquor is broken to flocculence when the particles of herbaceous matter, seized by those of the lime, and coalescing, appear large and flocculent; and the liquor interceding them is seen quite transparent when viewed by transmitted light in the narrow part of a wine-glass.

This breaking may also be distinguished in a bright silver spoonful of the liquor by reflected light.

On these grounds some lime ought to be added to canejuice which contains the ordinary quantity of herbaceous matter, not with the vain hope of separating all the herbaceous matter at once, but with the experienced certainty that the liquor yawed or cleansed with the aid of lime, will contain less herbaceous matter in solution than it would otherwise have retained, and will require the less additional lime to act on the melasses acid.

Towards the kind of depuration which can be effected in the process of yawing, lime thus contributes something, but not nearly so much as has been generally supposed : for a quantuy of lime which is sufficient to give a nauseous taste 
to the sugar, is yet incompetent to the extrication of all the herbaceous matter, so that it shall be separable by yawing or subsidence : and an excess of lime, not greater than $\frac{1}{500} \mathrm{dth}$ or $\frac{1}{3000} \mathrm{dth}$ of the weight of the juice, is constantly attended with a manifest debasement of the colour of the sugar, when this excess takes place in the beginning of the boiling, or previons to the reduction of the juice by evaporation. It is of no practical use to inquire after every agency by which the excess of lime has these effects; but it is expedient to observe, that when a juice is yawed with excess of lime, and cleared to transparency by subsidence, which soon takes place in a specimen quickly cooled in a wine-glass, it will show colour approaching to that of porter; while the like juice, treated in the same way, but with only a moderate dose of temper, will be almost colourless when transparent.

It is moreover to be observed in ordinary practice, that when too much temper has been used in the yawing, the liquor, during the boiling of the teaches, looks much browner than that which has been less tempered. The scum has a darker colour, and is more apt to break and sink into the liquor; and it has less of the tenacity and flocculence by which ordinary scum clings on the skimming instrument, and is separable by the common process.

The practical inference from all these facts is, that the temper ought to be used sparingly in the raw juice in the operation of yawing, although it should be found necessary to use more temper afterwards, for purposes different from those lately recited.

It is chiefly by reason of the agency of the temper on the melasses acid, or on that matter which most powerfully impedes the separation of the sugar from the mclasses motherliquor, that the temper is eminently useful in the manufacture of Muscovado sugar, and that greater quantities of it may be advantageously employed, provided the whole of it be not administered at once, and at the period of the manufacture in which it is apt to colour the juice, to lessen the buoyancy and tenacity of the scum, and to frustrate the labour of the skimmer. Among the signs which may guide 
an intelligent sugar-boiler in respect to the doses of temper, those which correspond with the foregoing observations are the simplest and most certain.

When the artist knows that the quantity useful in yawing is less than is necessary for a bold grain and speedy curing of the sugar, and is apprised of the practicability of abstracting all the temper that may be used afterwards in the teaches, he will not err by excess of temper in the first process.

Supposing that he tempers in a grand boiler, or uses a claxifier to the same effecl, he will introduce a quantity of temper rather too small than too great, when the vessel has received three-fourths of its charge. He will yaw and check the fire and skim, and then boil moderately and skim for eight or ten minutes. He will then take a wine-glass full of the liquor, and if, after cooling a little, it looks wheyey and not broken, he will use one-sixth or one-fourth of the first quantity of lime, and will immediately take a fresh sample. When the liquor appears broken, and clears with little or no colour, he has hit the precise quantity which is compatible with the cleanness and whiteness of the sugar, and which goes nearest to the whole quantity to be used for the perfection of the grain.

By the usual ladle-proof he will soon ascertain the additional quantity of temper to be used in the inspissated liquor, in the manner presently to be described.

He that will not follow this rule may take a grosser, by using three-fourths of the temper in the yawing, and the remainder in the inspissated liquor.

It has not been unusual for the boilers to put temper in the second and first teach, when the proof by the ladle showed that the temper used in yawing was not sufficient for the granulation of the sugar. Such temper there used was the least injurious to the colour of the sugar; but as none of it could now be separated by skimming from the thick syrup, it manifestly adulterated the sugar, and increased the quantity of filth to be seen in a solution of it.

But no such objection lies against the free use of temper in the syrup, provided we can extract all this temper and all the filth which it catches, and advance the syrup transparent 
and clear, to yield sugar emulating if not equalling ordinary coarse lump sugar, for general use, without abatement of the described celerity in the transition from the raw juice to sugar. For, in a just comparison of sugars to be used œeconomically, we are to advert, not merely to the degrees of whiteness, which may be varied by the size of the grain, but chiefly to the cleanness of equal solutions, and the sweetening effects of portions equal in dryness and in weight.

The common use of yellow sugar-candy, on the continent of Europe especially, shows that it is not the colour of good Muscovado sugar which has impeded the consumption of it in the crude state. It is truly the filth which appears in a solution of it, which has forbidden the culinary use of it, and depreciated it so far below lump sugar; and it is in consequence of the cleaning by filtration, rather than by reason of the blanching by breaking the grain and by claying, that the lump sugar has such preference and greater price.

It is this filth, also, which compels the sugar refiner in Europe to use ox blood in quantity proportionate to the inpurity of the Muscovado sugar; and to encounter the labour and waste attending the abstraction of the sweets from the abundant scum, consisting of the heterogeneous. matter entangled in the filaments of coagulated blood; and it is the chief cause of his inability to give a price for clry Muscovado sugar, nearer to that at which he sells the ordinary lump. sugar.

\section{Of the Improvement of Filtration.}

It will therefore be the first of all services for this island, and it will be the most desirable character of Janaica sugar, that it be made clean by the mere means of lime and filtration, which are employed in the manufacture of the finest lump sugar; and, until this character is established, little or nothing ought to be said concerning the other tempers and expedients which may be advantageously used in particular circumstances.

As the true meaning of the word flltration is not understood by all, and doubts and controversies may arise from the mere abuse or misconception of the term, it is expedient that it should be defined in a manner suitable to all the workmen, 
workmen, to whom these pages may ultimately be communicated.

When a foul liquor is passed throngh a fine wire sieve, or through a blanket, which stops the coarse filth, but not the finer, and where the liquor so treated still remains clouded or turbid, this is called straining : but when a medium, such as close cloth or unsized paper, is used, and this stops all extricated filth and passes the liquor transparent, the process is called fltration.

In the refineries in Europe a blanket serves as a Glter, but not before the filth of the solution of Muscovado sugar has been entangled in flocks of coagulated ox blood, too large to pass through the interstices of a blanket.

The use of such a woollen strainer has been tried in this island, but is generally abandoned for good reasons.

A blanket only strains the boiled cane-juice, while it passes with the velocity necessary for the manufacture of good Muscovado sugar. When the interstices of the blanket are narrowed by coarse floceulent filth, and when it begins to filtrate truly, the process becomes so stow to retard the manufacture intolerably : the last portions refuse to pass through; the quantity of sweets retained in and on a large thick blanket makes a great defalcation from the sugar, and the cleansing of the cloth is laborious.

To make the process of filtration applicable in the manufacture of Muscovado sugar, it was necessary that a medium should be employed capable of filtrating truly at the begin. ning. It was equally necessary that the velocity of this filtration should be eqqual to that with which the liquor would pass turbid through a coarse strainer of the same dimensions. It was moreover requisite, for the reasons above mentioned, that this rapid filtration should be confined to that period in which all the herbaceous matter is thrown out of solution, and in which the liquor is viscid with sugar.

These desiderata are attained in the invention lately presented under trial to as many of the honourable nembers of the appointed committees as could be assembled at the time and place.

In this first mechanism the proof of the principles and 
powers was chiefly attended to: in the instruments which are now to be made, the utmost convenience will be attained; and from these only the descriptions and models, which I shall soon lay before the bonourable members, are to be made.

At the present moment I must content myself with presenting a few lines descriptive of the principles upon which a small simple filtrating instrnment is competent to the intended urses.

In orkinary cases, the power by which the liquor is pressed through the interstices of a filtrating cloth is merely the weight of the liquor; and, under equal pressures by weight, the velocity of filtration is as the extent of filtrating cloth. But, as in ordinary vessels used for this purpose, the pressure on a given area of the filtrating surface increases with the height of the liquor above it, omitting nice discussions, we may affirm generally, that the velocity of filtration is as the number of square inches of filtrating cloth multiplied by the height of the contained pressing fluid; therefore, in order to attain any required velocity of filtration, the height of the pressing column may be made to compensate for any desirable reduction of the size of the cloth.

Supposing, for instance, a cloth measuring 8 feet by $7 \frac{7}{2}$, or 60 square feet in area, could filter fast enough under a charge of liquor measuring 3 inches in depth, one-sixth of this cloth, or 10 square feet of it, would serve if the column or charge of liquor pressing on it were made to be 18 inches in height: and with the height of 36 inches, 5 square feet would serve; and this is less than the measure of a common pocket bandkerchief.

By the like reasoning it will be found, that if the cloth of 60 square feet filter no quicker than is necessary for the progress of the manufacture, the filtration will become slower as the liquor lowers to a smaller area of filtrating surface, and still slower as the height of the pressing liquor decreases; that at the height of one inch, the quantity filtrated in a given time would be much less than one-third of that which the progress of the work would require.

But when it is considered that filtration will wet avail much 
much until the liquor is rich and viscid with sugar; that every filtration abates as the interstices of the cloth become narrowed, or clogged with filth; and that a rich saccharine liquor expanded on a large cloth in a basket, in the usual mode of filtration, will soon be chilled, and incapable of passing through the chilled and agglutinated filth; it will readily appear that the quantity of sweets retained in and upon the cloth would cause an enormous defalcation of the sugar, and great labour in washing out the sweets for the stillhouse, and the filth from any great filter. We are to revert, then, to the small filter above mentioned, acting by the pressure of a high column of liquor, in order to obviate the described inconveniences.

It may appear paradoxical at the first view, but it is a fact demonstrated in every school-book of natural philosophy, that the pressure of the liquor on the filter is the same whether the superincumbent column consists of a ton or of a pint of the liquor, provided the heights of these columns be equal.

Therefore, instead of making an instrument to give a ton of pressure, by containing about a ton, or 250 gallons of liquor, I have given an equivalent pressure by a pint or two contained in a slender perpendicular tube: and instead of setting the filtrating bag to a great capacity, I have set it vertical, flat, and with the opposite parallel sides so near to each other, as to leave room only for the filth to be collected from half a ton or a ton of sugar.

By such mechanism and pressure the juice may be filtrated truly and expeditiously, even when it is so rich and viscid with sugar that it could not pass through any filtrating medium used in the ordinary way. The filtration keeps pace with the ladling forward, when the cloth measures about 10 or 12 feet square, and the flattened bag about 5 square feet: and the same bag will clean from 10 to 15 or 20 hundred weight of sugar, according to the nature of the juice and the care of the workman in the previous depurations by yawing and skimming.

By these means, also, the whole quantity of liquor in the filter at any time may fall short of a gallon; and the quan- 
tity retained when the bag becomes stuffed and clogged with filth is too inconsiderable to deserve notice, even if the washings were not to go to the still-house. For the described pressure of the slender column of the liquor is, or may be, made competent to squeeze the filth with a force equal to that of one, two, or three tons weight, and to reduce it to the consistency of dough, if that were necessary.

The cloth which answers for this purpose by its closeness, and durability, and cheapness; is a kind of flannel called double swanskin: it is sewed to form a bag: the bag is placed flat between two reeded or fluted faces of wood: the mouth of the bag is perfectly closed, by giving it one fold or fell to be compressed by the reeded faces. The liquor is supplied from the slender pressing column by a small stopcock entering a small short tube of swanskin belonging to the bag : by drawing a little of this tube over its orifice, the ligature becomes closer as the pressure of the liquor becomes greater.

It is easy to conceive how quickly such a filter may be slung into its place or removed for washing, or to make way for another that is already washed.

To prevent fruitless experiments and expenses it is necessary to observe, that in any position different from the vertical now described, the bag will not act as a filter, and will only strain the liquor, and that, if the reeding be not vertical, the filtration will be slow.

When liquor is to be thrown forward, it is, in the general course of business, to be ladled from the second teach into the instrument, by the gutter, off which it will run clear and quickly into the first teach.

When the ladle is held in the usual method, the labour of throwing the liquor to the described height would be considerable: but it becomes uncommonly easy when the hook of a slender pendulous chain or rope is made to sling the handle of the ladle at a spur, marking the proper centre of motion, or the fulcrum on which the ladle is to play, with little effort to the workman.

At skipping-time the instrument reserves some filtered syrup to recruit the emptied teach : this is the business of a 
few minutes : if it were as many hours, such syrup would take no damage in the time.

About twelve ladlings forward belong to a skip; and for each of these its share of reserved temper is to be used. Less than a tea-spoonful generally serves : a small excess does no harm now to the colour of the sugar: both the filth and the temper are stopped in the filter; and the sugar becomes improved in the manner above described, provided no part of it be over-heated in the skipping.

The damage which the liquid sugar takes here is certainly greater, as it is fouler by the herbaceous matter; because the herbaceous matter becomes darkened or charred sooner than the mere sugar. But whether the filter be used or not, there will always be some depravation of the colour of the ground sugar when the skipping is conducted in the usual manner.

\section{Of the Spraying Instrument in Skipping.}

It is now sufficiently understood, that in boiling down and in ladling forward, great care ought to be taken that no part of a vessel, between the surface of the charge and the under-pinning, shall be so far heated as to burn-to the syrup or sugar adhering to it; and that this burning or charring is to be prevented by one negro rolling the residuary liquor up to the under-pinning, until another has ladled forward and charged the evacuated teach, and until the liquor froths to the under-pinning.

But as nothing of this kind is practicable in the skipping from the first teach, some new expedient is necessary to prevent the burning-to which is manifested in every whole skip, by the hissing heat of the copper, by the empyreumatic smell, and by the film of charred matter which washes from the sides into the subsequent charge of liquor; for the practice of some, in skipping only one-half of the ready and graining charge, is a very objectionable shift.

To preserve the crop and grain once attained, to prevent all empyreuma, and to maintain every advantage of the filtration, I have contrived, and presented in trial to the honourable 
nourable gentlemen of the committee, a simple instrument, which the negroes have since used with the greatest ease.

It is nothing more than a tube of timned iron or sheet copper, about six feet and 2 half in length, with a bore of one-half or three-fourths of an inch in diameter, delivering a spray of water on the bottom and sides of the skippingteach during the short time of skipping, and cooling it sufficiently and equally with a very small quantity of water.

Upon the common shed or arched covering which shelters the stoker, a cask of water is placed, and a slender hose or leathern tube inserted in the cask, delivers the high column of water into the metallic tube through a small stop-cock; and the other end of the metallic tube being provided with six small holes, like those in the top of a pepper-box, and looking upwards when the tube is held horizontal, the water is squirted in the form of spray by the heavy pressure of the high column to the extent of the bottom and sides of the teach only, and the spray ceases when the cock is closed.

The direction of the spray requires no skill of the negro; for a spur on the timned iron tube stops it at the proper place; and he has only to hold the tube straight forward, while he grasps it near the spur and near the mouth-piece of the fire-place with one hand, and holds or turns the cock with the other.

The fire is not disturbed, and no wet trash is allowed to chill the furnace and clot upon the grating. The spray evaporates as it touches and cools the teach; and as the fireface of the furnace remains untouched and red hot, the fresh charge boils in the first teach in half a minute after the spray is stopped, and the instrument now shut at the cock is withdrawn.

The advantage of this instrument is not confined to the prevention of empyreuma and colour; for it serves to make all the portions of the skipped mass equal in spissitude, or, as the workmen say, equally boiled, and enables the workman to defer the skipping until the proof is perfectly decisive; and these purposes are answered at the expenditure of two or three quarts of water. 
Sulservience of these Measures to the Improvement of Rum.

Concerning rum it is now to be observed, that it derives the depreciating characters of the recent spirit from two sources; the chief of which is the filth of the scums, and especially the first scums in yawing.

The tendency of such matter, even if there were nothing verminous or animalcular in it, is to the putrefactive fermentation, or rotting, while that of the sweet is to the vinous fermentation, and thence to the acetous; the product of the former fermentation is as offensive to the smell and taste, and as noxious, as that of the latter is grateful and cordial. Wherever scums are detained to await the spontaneous separation of the sweets from the filth, an intestine motion may be observed, and then chiefly in the concurrence of these fermentations the offensive product is generated; the rest is formed in the fermenting vats, in quantity proportionate to the filth of this kind which passes into them.

Every vinous liquor capable of yielding an intoxicating spirit by distillation, affords some quantity of peculiar essential oil, which awaits the arise of the water of the latter and weaker runnings, and characterizes them; therefore this essential oil is, in a great measure, separable from the spirit by redistillation; especially if salts retentive of the water, and restraining the volatility of the oil, are used.

But it is peculiar to the ordinary manufacture of rum that very offensive ethereal fluid is generated in these mixed fermentations, and that by reason of its volatility it is inseparable by a redistillation.

But from the source above mentioned the essential oil of rum acquires extraordinary nauseousness; and as a single redistillation cannot exclude it totally, and as any number could not exclude the ethereal taint above mentioned, the best new rum of any estate is that which runs intermediate in respect of the offensive ether and the fetid oily feints:

All rum is improved by time in wooden casks, by exhalation of ether and absorption of oil, and under a growing charge for waste and for interest on the price. Some bave improved it sooner by ventilation, but not without a great

Vol. 24, No. 96. May 1806. X

waste 
waste of spirits; but now it may be remarkably improved immediately by measures which prevent the above contamination; and the first of these is the abstraction of the putrefactive matter by filtration, and the immediate conveyance of the clear warm fragrant liquor to the working cistern, there to undergo the most timely and productive fermentation, and to suffer the least defalcation of spirit by foul scum and bottoms, which are generally thrown away.

Another source of the contamination is in the empyreuma; but as this regards the distillation as well as the errors in making sugar, it is unnecessary to say more of it at present than that the pressribed measures, together with a judicious setting and management of the still, will totally prevent the empyreumatic smell and taste.

LVII. Letter of M. TARDY DE LA Brossy to Professor PicteT, of Gcneva, upon the Experiments of Mr. BiDDLE, relative to the Density of frozen Mercury*.

SIR,

Joyeuse (A rdêche), Oct. 13, 1805.

T HE experiments of Mr. Biddle (vol. xxxix. p. $217+$ ), in order to determine the density of solid mercury, having attracled my attention, I cannot pass over in silence the serious objections to which the results of the above gentleman are liable. In communicating them to you, I am well persuaded that I shall enter completely into your views, which have equally for their objects the extension of truth and the removal of error.

Mr. Biddle, after having made known the process which he followed and the precautions taken by him, informs us, that a thousand grains of mercury made solid at the 40th degree below the zero of Fahrenheit ( -32 of Reaumur), experienced, when weighed in alcohol of the same temperature, a loss in weight of $59^{\circ} 8$ grains.

A thousand grains of pure silver, weighed by the same

* From Billiotheque Britannique, vol. xxx.

+ Vide Nicholson's Journal for April 1805.

balance, 